\title{
Therapeutic Effect of Balloon-occluded Retrograde Transvenous Obliteration on Portal-systemic Encephalopathy in Patients with Liver Cirrhosis
}

\author{
Tomohiro Kato, Takahiro Uematsu, Yoichi Nishigaki*, Jun-ichi Sugihara*, \\ Eiichi TOMITA* and Hisataka MoRIWAKI
}

\begin{abstract}
Objective Balloon-occluded retrograde transvenous obliteration (B-RTO) has recently been introduced as a new interventional modality to prevent fatal bleeding from solitary gastric varices. A large portal-systemic shunt including gastric varices also causes severe encephalopathy in some cirrhotic patients. In this study, we evaluated the effect of B-RTO as a candidate therapeutic method to treat chronic recurrent hepatic encephalopathy due mainly to a portal-systemic shunt.

Patients and Methods Since July 1995, we experienced 43 cirrhotic patients with chronic reccurent hepatic encephalopathy. Among them, six patients had anigographically proven large ( $>1 \mathrm{~cm}$ in diameter) portal-systemic shunt, and received B-RTO. B-RTO was carried out only once using $5 \%$ ethanolamine oleate with iopamidole to obliterate the portal-systemic shunt for 30 minutes. The median observation period after B-RTO was 29 months (range 23-46 months).

Results In all 6 patients, encephalopathy had disappeared after B-RTO, and the patients were free of encephalopathy during the following 6 months. B-RTO significantly reduced blood ammonia levels at one month, 3 months, and 6 months later, without affecting serum aspartate aminotransferase activity, total bilirubin and albumin concentrations, and plasma prothrombin time. Encephalopathy relapsed in 4 patients between 6 and 30 months. Additional B-RTO was required and effective in 2 of them.

Conclusion B-RTO is an effective treatment for chronic recurrent hepatic encephalopathy with an angiographically proven portal-systemic shunt.
\end{abstract}

(Internal Medicine 40: 688-691, 2001)

Key words: hepatic encephalopathy, portal-systemic shunt, interventional radiology

\section{Introduction}

It is well known that patients with liver cirrhosis experience many complications including variceral bleeding, hepatic encephalopathy, ascites, jaundice, and renal dysfunction (1). As variceral bleeding is fatal, many therapies have been developed such as endoscopic sclerotherapy (EIS) with 5\% ethanolamine oleate or endoscopic variceral ligation therapy (EVL) $(2,3)$. Although EIS and EVL are very effective and have decreased the death rate of the patients with esophageal varices $(2,3)$, such therapies have limitations in the use for treatment of cardiac varices. Instead of endoscopic modalities, interventional radiology represented by balloon-occluded retrograde transvenous oblitation (B-RTO) has recently been described as a new therapy for solitary gastric varices (4).

On the other hand, encephalopathy in cirrhotic patients is not easily treatable. There are two types of encephalopathies in liver cirrhosis; portal-systemic encephalopathy and end-stage hepatic encephalopathy in severe liver dysfunction (1). Portalsystemic encephalopathy is much different from the latter encephalopathy in the pathogenesis. The portal-systemic shunt involves a blood flow from mainly the supramesenteric vein to the systemic vein, and provides high systemic blood ammonia levels. Solitary gastric varices are constructed with spleno-renal shunt, and have also been reported to cause severe encephalopathy in some patients. It has been preliminarily reported that patients who had underwent B-RTO for cardiac varices were sometimes free of encephalopathy thereafter. Such cases suggest that B-RTO occluded the portal-systemic shunt, which might have been a cause of varices as well as encephalopathy. In light of this, a hypothesis can be further expanded that BRTO is a candidate treatment for a chronic portal-systemic encephalopathy in cirrhotic patients regardless of the presence of cardiac varices.

To test this hypothesis, we evaluated the effect of B-RTO on chronic recurrent hepatic encephalopathy in cirrhotic patients

From the First Department of Internal Medicine, Gifu University School of Medicine, Gifu and *the Department of Gastroenterology, Gifu Municipal Hospital, Gifu

Received for publication August 30, 2000; Accepted for publication January 16, 2001

Reprint requests should be addressed to Dr. Hisataka Moriwaki, the First Department of Internal Medicine, Gifu University School of Medicine, 40 Tsukasamachi, Gifu 500-8705 


\section{B-RTO for Portal-systemic Encephalopathy}

who had angiographically proven portal-systemic shunt without varices.

For editorial comment, See p 677.

\section{Patients and Methods}

Since July 1995 , we have treated 43 cirrhotic patients with chronic recurrent hepatic encephalopathy. Among them, six patients (three males and three females) were enrolled in this study after fulfilling the inclusion criteria of both the resistance to conventional treatment of hepatic encephalopathy and the presence of angiographically proven portal-systemic shunt. Their age, sex, serum hepatitis virus markers, and modified Child's classification are given in Table 1 . They had been admitted to our hospital at least 3 times or more due to chronic recurrent encephalopathy of grade III, and were treated at each episode with drip infusion of branched-chain amino acid enriched solution. They had been also treated with oral lactulose throughout both the in- and outpatient period. We then checked for the existence of portal-systemic shunts using magnetic resonance angiography (MRA) and dynamic X-ray computed tomography (CT), and identified a variety of portal-systemic shunts in all patients (Table 1). The diameter of the shunts ranged from $1.0 \mathrm{~cm}$ to $2.0 \mathrm{~cm}$. Gastro-esophageal varices was found in only one patient (Patient No. 6).

B-RTO was carried out only once in patients No. 1-5 according to the method of Kanagawa et al (4) using 5\% ethanolamine oleate with iopamidole (5\%EO) to obliterate the portal-systemic shunt for 30 minutes. Patient No. 6 received BRTO three times to simultaneously treat her solitary gastric varices. The volume of infused 5\%EO ranged from 10 to $14 \mathrm{ml}$ (Table 1). Median observation period after B-RTO was 29 months (range 23-46 months) (Table 1). During this period, we monitored the patients by physical examination and blood biochemistry analysis basically every 2 weeks, and with MRA and dynamic CT every 3 months.

This study was approved by each institution's review board for human research. Written informed consent was obtained from each patient or his/her relatives in advance of B-RTO.

\section{Results}

Complete obliteration was achieved without major complications such as development/enlargement of esophago-cardiac varices or increase in ascites. Varices of Patient No. 6 disappeared. Encephalopathic symptoms had disappeared clinically in all 6 patients within 2 days after B-RTO. Thereafter, they continued to receive same medical treatments other than branched-chain amino acid enriched solution. They were free of encephalopathy during the following 6 months (Fig. 1).

Patient No. 1 relapsed into grade III hepatic coma after 18 months. He was again administered B-RTO for the same portal-systemic shunt, and survived without hepatic coma until he died from hepatocellular carcinoma at 30 months. Patient No.

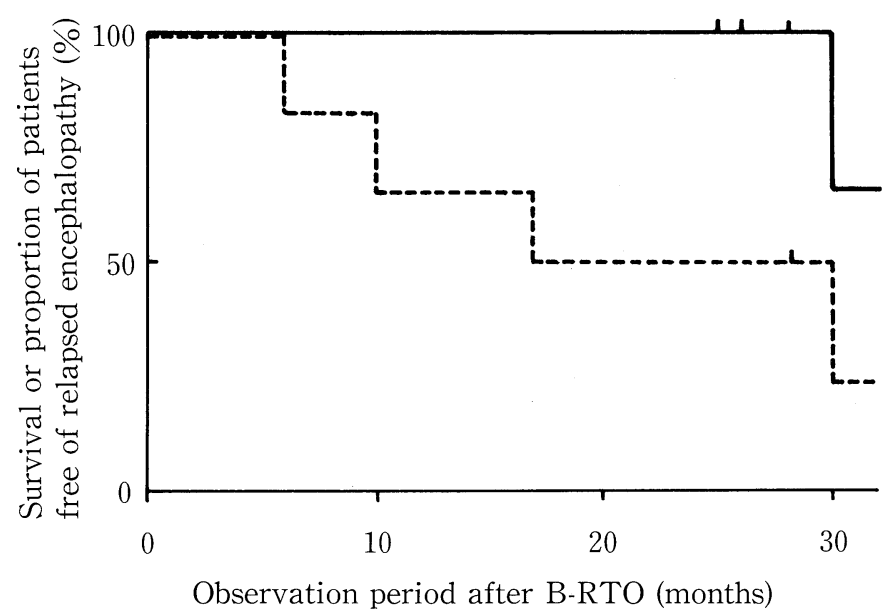

Figure 1. Overall survival (solid line) and proportion of patients free of relapsed encephalopathy (dotted line) after balloon-occluded retrograde transvenous obliteration (B-RTO) for portalsystemic encephalopathy in liver cirrhosis $(n=6)$.

Table 1. Clinical Characteristics of Patients Treated with B-RTO

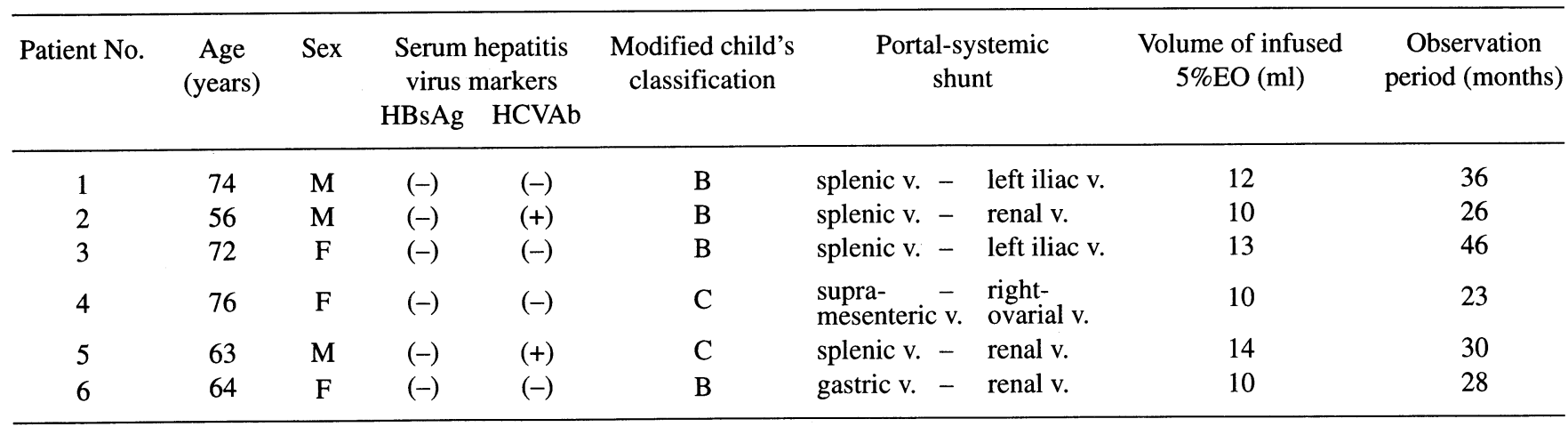

B-RTO: balloon-occluded retrograde transvenous oblitation, EO: ethanolamine oleate, M: male, F: female. All patients were negative for anti-nuclear antibody or anti-mitochondrial antibody. 

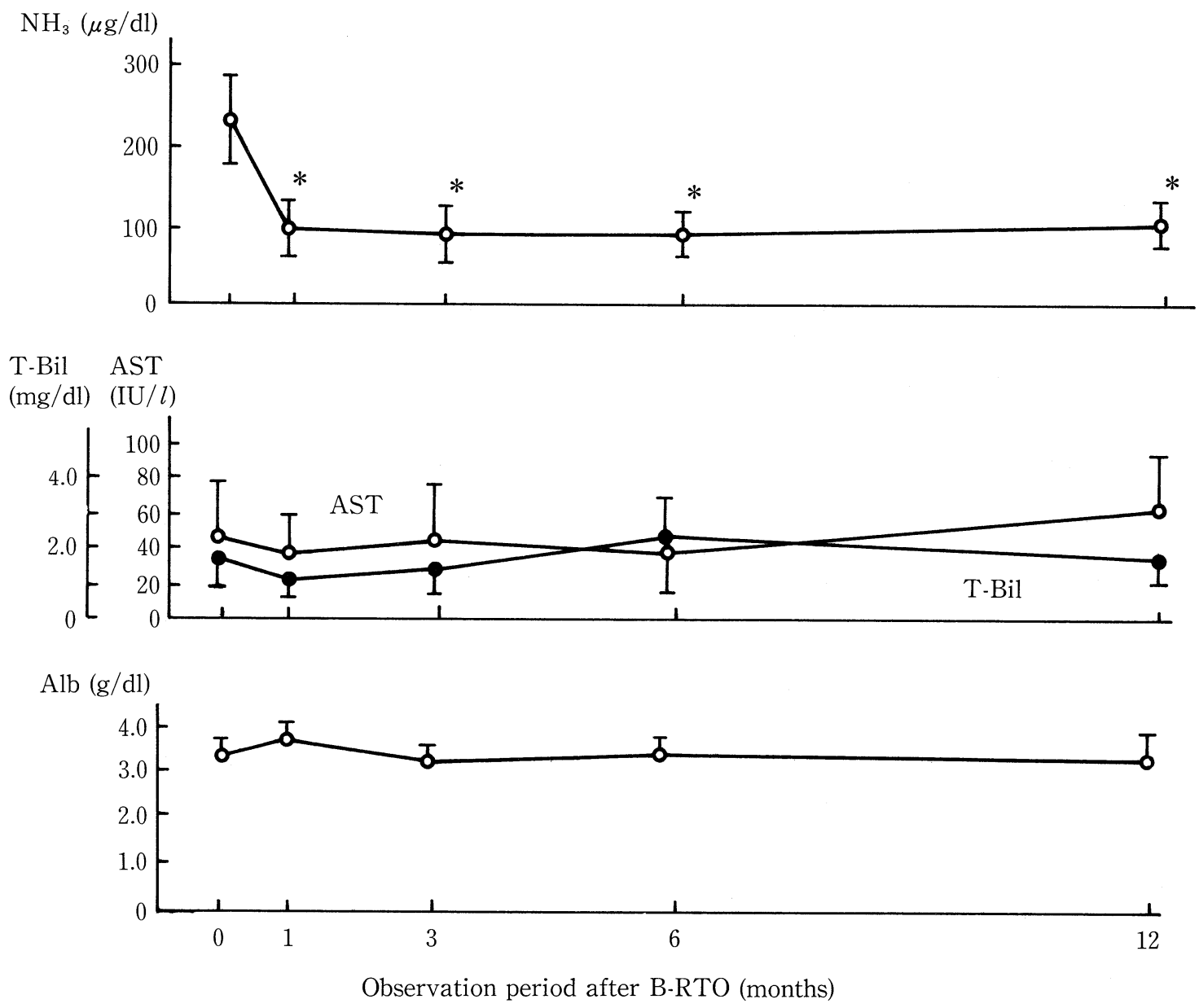

Figure 2. Time courses of changes in blood ammonia level $\left(\mathrm{NH}_{3}\right)$, serum aspartate aminotransferase activity (AST), and total bilirubin (T-Bil) and albumin (Alb) concentrations after balloon-occluded retrograde transvenous obliteration (B-RTO) for portal-systemic encephalopathy in patients with liver cirrhosis. Values are expressed as mean and standard deviation $(n=6) .{ }^{*} \mathrm{p}<0.05$ as compared to the value before B-RTO ( 0 month) by the paired $t$ test.

4 had also grade III hepatic coma after 10 months. She was also readministered B-RTO, and was free of encephalopathy in the remaining observation period. Patient No. 2 and Patient No. 5 experienced hepatic coma of grade II 30 and 6 months later, respectively. In these 2 patients, possibly responsible portal-systemic shunt could not be found by MRA or dynamic $\mathrm{CT}$ at the relapse episode. They were effectively treated using branched-chain amino acid enriched solution. Patient No. 3 and Patient No. 6 were free of hepatic coma for the entire observation periods of 46 months and 28 months, respectively. Overall survival after B-RTO for these 6 patients is given in Fig. 1.

Blood ammonia levels were significantly decreased one month after B-RTO as compared with those before B-RTO, and remained low up to 12 months (Fig. 2). B-RTO did not affect serum aspartate aminotransferase activity, total bilirubin and albumin concentrations (Fig. 2), or plasma prothrombin time.

\section{Discussion}

Chronic recurrent hepatic encephalopathy is often associated with portal-systemic shunt in cirrhosis patients (5). We usually treat hepatic encephalopathy of this type with lactulose and oral branched-chain amino acid supplementation (6). In general, these therapies are effective to improve each episode and to prevent recurrence of hepatic encephalopathy. However, we sometimes experience a limitation in therapeutic as well as preventive effects of these modalities in a population of patients with portal-systemic shunt, and in such particular patients, repeat encephalopathic episode occurs quite frequently. Although the incidence of these resistant cases has not ever been described, the present study provides an estimation that they account for approximately $14 \%$ (6/43) of chronic recurrent hepatic encephalopathy.

On the other hand, many cirrhotic patients have esophago- 
cardiac varices. When variceral bleeding impends, these patients undergo endoscopic therapies such as sclerotherapy (3) or ligation (2). However, it is difficult to treat solitary gastric varices with EVL or EIS. To overcome such limitations of endoscopic modalities, interventional radiology including balloonoccluded retrograde transvenous obliteration (B-RTO) has been recently introduced for clinical use to treat solitary gastric varices (4). In addition to excellent hemostatic effects of B-RTO (4), it has also been reported that a patient, who had received B-RTO for varices, was free of encephalopathy for a short period following the procedure (7). The effectiveness of B-RTO depends basically on its ability to obstruct portal-systemic shunt. Therefore, it can be hypothesized that B-RTO will be a possible new therapy for portal-systemic encephalopathy even in patients without cardiac varices. We demonstrated this hypothesis in 6 patients by significantly reducing the blood ammonia level and eradicating encephalopathy. Possible adverse effects such as esophago-gastric varices and ascites were not induced by B-RTO. Another merit of B-RTO was that the method did not affect the functional reserve of the liver.

Although several other methods are available to obstruct portal-systemic shunt including surgical ligation, a surgical approach may cause too great of a load on severe cirrhosis. Transjuglar intrahepatic portal-systemic shunt (TIPS) is one of the more useful therapies for the prevention of recurrent variceral bleeding, and has also been reported as a useful therapy for chronic hepatic encephalopathy (8). TIPS has another benefit to avoid the risk of developing refractory ascites due to shunt occlusion. However, TIPS seems hardly applicable to severe atrophic cirrhosis caused by viral hepatitis. Hence, when patients experience liver atrophy and spleno-renal shunt, BRTO is a safer and less invasive method, and TIPS should be selected as a second choice to treat hepatic encephalopathy in particularly. Transjugular obliteration (TJO), a method similar to B-RTO, has been reported as an effective approach to portal-systemic shunts as well (9), but requires great skill.

We treated cirrhosis patients who experienced portal-systemic encephalopathy without esophagocardiac varices only once by B-RTO at first, while several sessions of B-RTO are usually required to obliterate varices as in Patient No. 6. Single session B-RTO seems sufficient to prevent recurrent encephalopathy up to 18 months (median), but might be feasible for recirculation of the shunt. Another benefit of the single session B-RTO is the lack of complications such as ascites, which are often observed after multiple sessions of B-RTO (4). As to the duration of the effect on hepatic encephalopathy, other interventional radiological therapies for shunt occlusion are limited to within one year $(7,8)$. However we cannot assert that the single B-RTO is more beneficial than multiple administrations for hepatic encephalopathy in terms of the effective period and the incidence of adverse effects. We suggest the necessity of further study to clarify this concern.

As to the machanism of the effect of B-RTO on portal-systemic encephalopathy, single session B-RTO as described in this study might have decreased the shunt flow and recovered the hepatic blood flow. It is considered that the decrease of blood ammonia level was brought about by the recovered hepatic blood flow and by the enhanced ammonia detoxication by the liver. The Child's classification in two-thirds of our cases was B, which suggests another condition for recovery from hepatic encephalopathy with single session B-RTO, i.e. the functional reserve of the cirrhotic liver is better conserved to process the increased ammonia load.

In conclusion, B-RTO is an effective treatment for chronic recurrent portal-systemic encephalopathy. Candidates for BRTO should be defined more precisely by a future prospective study.

\section{References}

1) Sherlock S. Hepatic Cirrhosis. Diseases of the Liver and Biliary System (Eighth Edition). Blackwell Scientific Publications, London, 1989: 410424.

2) Saeed ZA, Stiegmann GV, Ramirez FC, et al. Endoscopic variceal ligation is superior to combined ligation and sclerotherapy for esophageal varices. Hepatology 25: 71-74, 1997.

3) Kiviniemi H, Makela J, Haukipuro K, Kairaluma MI. Injection sclerotherapy for oesophageal varices: a ten-year experience. Hepato-Gastroenterology 42: 546-550, 1995.

4) Kanagawa H, Mima S, Kouyama H, Gotoh K, Uchida T, Okuda K. Treatment of gastric fundal varices by baloon-occluded retrograde transvenous obliteration. J Gastroenterol Hepatol 11: 51-58, 1996.

5) Bustamante J, Rimola A, Ventura PJ, et al. Prognostic significance of hepatic encephalopathy in patients with cirrhosis. J Hepatol 30: 890-895, 1999.

6) Yoshida T, Muto Y, Moriwaki H, Yamato M. Effect of long-term oral supplementation with branched-chain amino acid granules on the prognosis of liver cirrhosis. Gastroenterol Jpn 24: 692-698, 1989.

7) Ohmoto K, Miyake I, Tsuduki M, Ohno S, Yamamoto S. Control of solitary gastric fundal varices and portosystemic encephalopathy accompanying liver cirrhosis by balloon-occluded retrograde transvenous obliteration (B-RTO): a case report. Hepato-Gastroenterology 46: 1249-1252, 1999.

8) Luca A, D'Amico G, La Galla R, Midiri M, Morabito A, Pagliaro L. TIPS for prevention of recurrent bleeding in patients with cirrhosis: meta-analysis of randomized clinical trials. Radiology 212: 411-421, 1999.

9) Chikamori F, Shibuya S, Takase Y, Ozaki A, Fukao K. Transjugular retrograde obliteration for gastric varices. Abdom Imaging 21: 299-303, 1996. 